\title{
Complicações do uso de haste intramedular bloqueada no tratamento de fraturas de fêmur
}

\author{
Complications of use locked intramedullary nail in \\ the treatment of fractures of femur
}

\author{
Maxsuel Fidelis de Pádua Almeida', Thales Carneiro Farias², \\ João Bruno Ribeiro Machado Lisboa²
}

Almeida MFP, Farias TC, Lisboa JBRM. Complicações do uso de haste intramedular bloqueada no tratamento de fraturas de fêmur / Complications of use locked intramedullary nail in the treatment of fractures of femur. Rev Med (São Paulo). 2012;91(4):267-71.

RESUMO: As fraturas diafisárias do fêmur são frequentemente graves e decorrem de acidentes de alta energia, que podem acometer outros órgãos. A melhor abordagem terapêutica para as fraturas diafisárias do fêmur é eminentemente cirúrgica. $\mathrm{Na}$ atualidade muitos ortopedistas e traumatologistas preferem a opção terapêutica por hastes intramedular, que é um dos exemplos de fixação biológica, que por apresentar um procedimento com técnica cirúrgica simples e padronizada, contribui para uma melhor estabilização das fraturas com um mínimo de manipulação dos fragmentos a fim de manter sua vascularização, menores danos aos tecidos moles e o potencial de consolidação ser maior e permitindo assim uma carga precoce. Mas isto não a isenta de complicações, sendo a infecção pós-operatória a mais temida. Objetivo: Analisar as publicações científicas sobre as complicações que mais acometem os pacientes submetidos a esta técnica cirúrgica. Metodologia: Revisão da literatura através de uma pesquisa bibliográfica, localizados através da Biblioteca Virtual em Saúde (BVS) Resultado: Foram avaliados 20 artigos científicos e, observamos um total de 326 pacientes. Onde encontramos 215 complicações, destas complicações a anisomelia tem uma maior incidência $(50,70 \%)$; falha na consolidação vem em seguida com (17,21\%); infecção de partes moles (6,98\%); limitação do arco do movimento do joelho $(5,58 \%)$; desvio rotacional (4,65\%); desvio angular (4,19\%); inserção da haste na bursa $(2,33 \%)$; embolia gordurosa $(1,86 \%)$; pneumonia $(1,40 \%)$, dor $(0,93 \%)$, infecção da ferida operatória $(0,93 \%)$ e embolia pulmonar $(0,93 \%)$, osteomielite $(0,47 \%)$, necrose epifisária $(0,47 \%)$, TVP $(0,47 \%)$, insuficiência respiratória $(0,47 \%)$ e perfuração do córtex do colo femural $(0,47 \%)$. Conclusão: Foi observado um número muito reduzido nas complicações com esta técnica, não considerando qualquer outro fator que venha a desencadeá-la. Nas próximas pesquisas deverão ser analisados se estes valores são referentes a técnica realizada ou a diminuição dos fatores de risco para a realização do procedimento cirúrgico.

DESCRITORES: Fraturas do fêmur/complicações; Fixação intramedular de fraturas/métodos; Diáfises/lesões; Fraturas ósseas; Literatura de revisão como assunto.

\begin{abstract}
The femoral shaft fractures are frequent and often serious accidents result from high-energy, which can affect other organs. Nowadays many orthopedists and traumatologists prefer therapeutic option for intramedullary rods, which is an example of fixation, which present a procedure with a simple surgical technique, standardized, contributes to better stabilize the fracture with minimal manipulation of the fragments order to maintain its vascularization, reduced damage to soft tissues and the potential for consolidation be greater, thus allowing an early load. But this is not free of complications, and postoperative infection the most feared. Objective: To analyze the scientific publications about the complications that most affect patients undergoing this surgical technique. Methodology: Literature review through a literature search. located through the Virtual Health Library (VHL) Result: A total of 16 scientific papers and observed a total of 326 patients. Where we found 215 complications, these complications anisomelia has a higher incidence $(50.70 \%)$, fails to consolidate follows with (17.21\%), soft tissue infection (6.98\%), limited arc of motion knee (5.58\%); rotational deviation (4.65\%), angular deviation (4.19\%), insertion of the rod in the bursa (2.33\%), fat embolism (1.86\%) and pneumonia $(1.40 \%)$, pain $(0.93 \%)$., wound infection $(0.93 \%)$. and pulmonary embolism $(0.93 \%)$, osteomyelitis $(0.47 \%)$, epiphyseal necrosis $(0.47 \%)$, DVT $(0.47 \%)$, respiratory failure $(0.47 \%)$ and perforation of the cortex of the femoral neck $(0.47 \%)$. Conclusion: We found very few complications with this technique in not considering any other factors that will unleash it. In future research were to be analyzed if these values are related to the technique performed, or the reduction of risk factors for the surgical procedure.
\end{abstract}

KEYWORDS: Femoral fractures/complications; Fracture fixation. intramedullary/methods; Diaphyses/injuries; Fractures, bone; Review literature as topic.

1. Graduando em Medicina na Faculdade de Medicina Nova Esperança (FAMENE) - João Pessoa, PB - Brasil.

2. Graduando em Medicina na Faculdade de Medicina Nova Esperança (FAMENE).

Endereço para correspondência: Maxsuel Fidelis de Pádua Almeida. Rua Antônio de Souza Leão, n. 156, Jardim Oceania - João Pessoa, PB. CEP: 58037-418. E-mail: maxsuel_17@ hotmail.com 


\section{INTRODUÇÃO}

As fraturas do fêmur são frequentes, geralmente são graves e decorrem de acidentes de alta energia, que podem acometer outros órgãos. O fêmur é o osso maior e mais forte do esqueleto humano e possui um envoltório muscular bem vascularizado, que promove rápida consolidação das fraturas, na maioria dos pacientes ${ }^{1}$. O tratamento das fraturas diafisárias do fêmur é eminentemente cirúrgico ${ }^{2}$, onde a Haste intramedular é a mais indicada ${ }^{3}$, desde o ponto de vista anatômico,funcional e fisiológico ${ }^{6}$, porém á complicações consideráveis, onde a mais temida seria a infecção, todavia esta não é a mais frequente ${ }^{3}$.

A menor manipulação desse invólucro ao redor do osso tem importância na manutenção da irrigação dos fragmentos e no processo biológico da consolidação óssea. Esse é o conceito da fixação biológica em que se estabilizam as fraturas com um mínimo de manipulação dos fragmentos a fim de manter sua vascularização e o potencial de consolidação, um dos grandes exemplos de fixação biológica seria a haste intramedular ${ }^{4}$.

Em 1958, o grupo AO na Suíça, sob a influência da ortopedia belga e com o apoio do Seguro Social do país, demonstrou que o tratamento cirúrgico das fraturas diafisárias do fêmur em humanos poderia oferecer melhores resultados funcionais, com redução do tempo de afastamento das atividades profissionais e das indenizações por incapacidade, a haste intramedular tem um objetivo de ser resistente o suficiente para suportar carga sem que haja intervenção no foco da fratura ${ }^{5}$.

As principais hastes eram introduzidas sem dilatação do canal medular, mas as observações clínicas logo mostraram que a estabilidade conseguida era, muitas vezes, insuficiente principalmente para controlar os desvios rotacionais. Para contornar esse inconveniente foi desenvolvida a técnica de fresagem, o que conferia melhor estabilidade à montagem e à instalação das hastes. Assim, foi possível tratar fraturas mais complicadas, conseguindo-se estabilidade suficiente para permitir o apoio precoce do membro ${ }^{7}$.

No Brasil surgiram alguns problemas relacionados à utilização de hastes intramedulares bloqueadas, devido aos elevados custos financeiros dos instrumentos das hastes e a necessidade de intensificadores de imagem ${ }^{4}$. Porém, na atualidade, muitos ortopedistas e traumatologistas optam pela conduta terapêutica por hastes intramedulares, devido sua consolidação ser maior comparada com outros tipos de sínteses ${ }^{8}$.
Atualmente, preconiza-se que o tratamento e fixação de fraturas sejam realizados com o método chamado de "fixação biológica", onde a manipulação e o trauma no foco de fratura são os menores possíveis. Sendo a haste intramedular bloqueada o maior exemplo promovendo uma satisfatória estabilidade na linha de fratura e suportar cargas elevadas in vivo, o que permite ao paciente retornar da anestesia com o apoio do membro fraturado 9 .

\section{OBJETIVO}

Desenvolvemos este trabalho com o intuito de analisar as publicações científicas, sobre a incidência de complicações que acometem os pacientes submetidos à colocação de haste intramedular em cirurgias de fratura do fêmur.

\section{METODOLOGIA}

Devido a haste intramedular ser o tratamento mais utilizado na fratura de fêmur, onde o mesmo é uma das principais fraturas que ocorrem em traumas de alta energia e um dos grande exemplos são acidentes automobilístico, que ocorrem em cidades de pequeno, médio e grande porte populacional,fato este que chama a atenção por ser um procedimento bastante utilizado, porém apresentando uma margem de complicações muito próximas do sucesso terapêutico.

Com as várias opções que dispomos para gerar conhecimento no âmbito da medicina, no desenvolvimento deste estudo, optou-se por uma revisão bibliográfica através de pesquisas publicadas em artigos nas revistas eletrônicas especializadas, localizados através da Biblioteca Virtual em Saúde (BVS), PubMed, EMBASE, com a análise de 20 artigos nacionais, sendo pesquisados os descritores referentes as fraturas femorais, seu principal tratamento e as complicações do tratamento mais utilizado, no período de maio a julho de 2012.

Atendo-nos ao caráter da temática, realizamos uma análise crítica e ampla das publicações correntes a partir de uma revisão bibliográfica não sistemática como estratégia de apensar informações e conceitos ao presente estudo.

\section{RESULTADOS}

Foram avaliados 20 artigos científicos e deles obtidos os dados sobre as complicações em pacientes que realizaram tratamento cirúrgico para correção de fraturas do fêmur, observando um total de 326 pacientes. 
O perfil epidemiológico dos pacientes que possuíram algum tipo de complicação ficou impossibilitado devido algumas fontes bibliográficas não detalharem os dados necessários dos pacientes que possuíram algum tipo de complicação, após o ato cirúrgico.

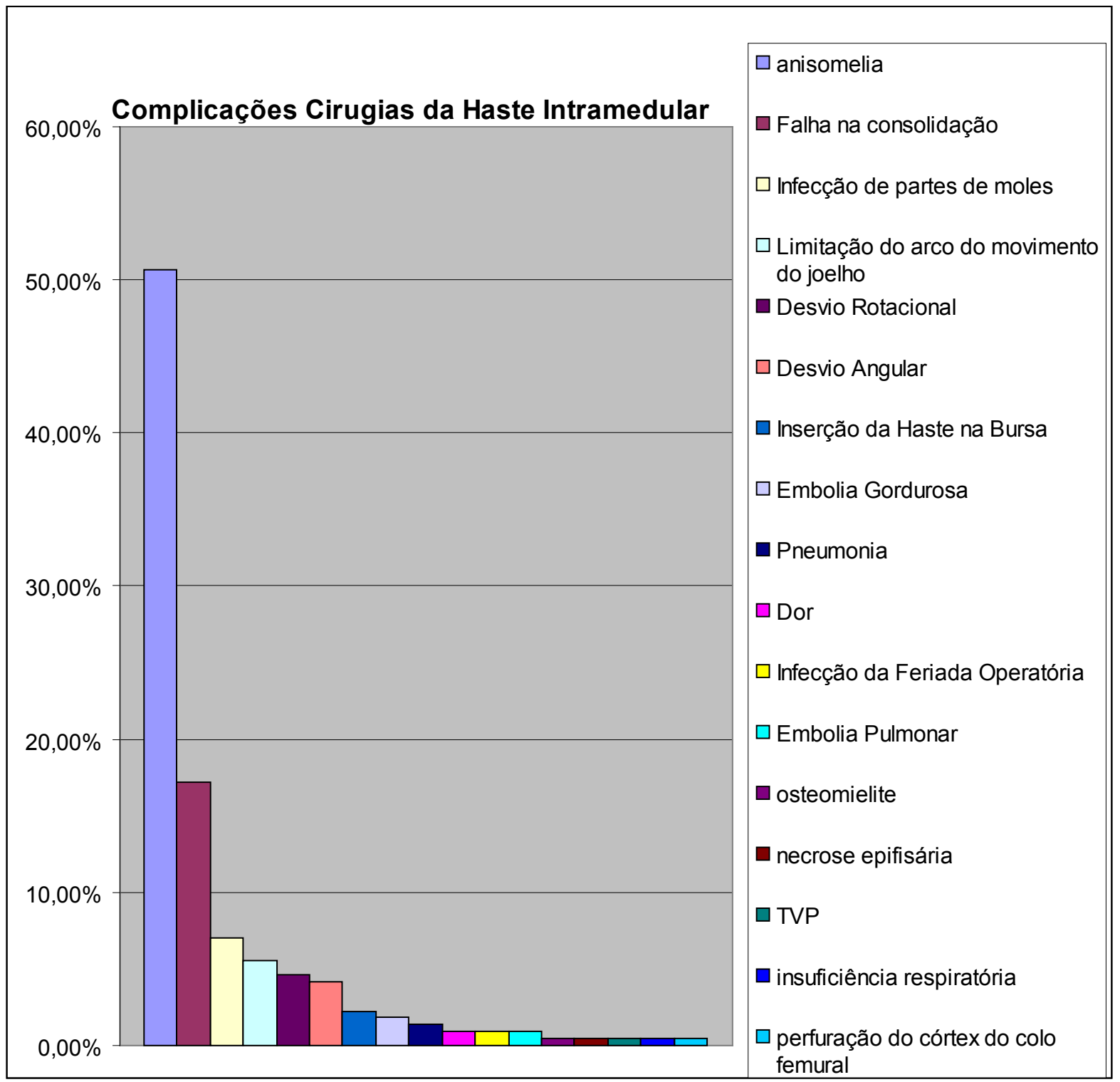

Encontramos 215 paciente com complicações, destas complicações a anisomelia tem uma maior incidência $(50,70 \%)$; falha na consolidação vem em seguida com (17,21\%); infecção de partes moles (6,98\%); limitação do arco do movimento do joelho (5,58\%); desvio rotacional (4,65\%); desvio angular $(4,19 \%)$; inserção da haste na bursa $(2,33 \%)$; embolia gordurosa $(1,86 \%)$; pneumonia $(1,40 \%)$, dor $(0,93 \%)$, infecção da ferida operatória $(0,93 \%)$, e embolia pulmonar $(0,93 \%)$, osteomielite $(0,47 \%)$, necrose epifisária $(0,47 \%)$, TVP $(0,47 \%)$, insuficiência respiratória $(0,47 \%)$ e perfuração do córtex do colo femural $(0,47 \%)^{10-18}$.

\section{DISCUSSÃO}

A haste intramedular sem duvidas é um grande avanço da medicina ortopédica, nas fraturas de fêmur mais especificamente falando.

Quando a mesma é indicada de forma correta como por exemplos: (falha em se conseguir uma redução aceitável por meios fechados; lesões 
traumáticas múltiplas associadas; lesão da artéria femoral coexistente necessitando de reparo; paciente idoso par aquém o repouso em cama prolongado é deletério; fraturas patológicas), a uma boa resposta terapêutica, porém os mesmo pacientes não estão isentos de complicações ${ }^{3}$.

A haste intramedular obtêm um leve grau de vantagem sobre as outras técnicas usadas, como podemos ver no tratamento não-operatório tem como inconvenientes o prolongado tempo de hospitalização e tratamento, a maior evidência de rigidez articular e consolidação viciosa. A osteossíntese com placa e parafusos necessita de maior agressão às partes moles, com maior desperiostização do osso, aumentando-se o risco de infecção e incidência de retarde de consolidação, com consequente fadiga do material de síntese ${ }^{20}$.

Já na osteossíntese com haste intramedular permite o realinhamento do osso, a rápida regeneração e união da fratura e o uso funcional precoce do membro ${ }^{10}$. Podemos citar como complicações locais: anisomelia, falha na consolidação, infecção de partes moles, limitação do arco do movimento do joelho, desvio rotacional, desvio angular, inserção da haste na bursa, infecção da ferida operatória, osteomielite, necrose epifisária e perfuração do córtex do colo femural. Também encontramos complicações sistêmicas: embolia gordurosa, pneumonia, dor, embolia pulmonar,TVP e insuficiência respiratória ${ }^{10-18}$.

A complicação mais encontrada é a discrepância de comprimento, ou anisomelia, é definida como uma condição na qual os membros emparelhados são visivelmente desiguais. Quando

\section{REFERÊNCIAS}

1. Guerra MTE, Bruch A, Bigolin AV, Souza MP, Echeveste $S$. Evolução clínica de pacientes operados por fraturas diafisárias do fêmur em um serviço especializado. Um estudo prospectivo.Rev AMRIGS. 2010;54(3) [citado 9 maio 2012]. Disponível em: www. amrigs.com.br/revista/54-03/009-612_evolucao\%20 clinica.pdf.

2. Pires RES. Fratura diafisária do fêmur: reprodutibilidade das classificações AO-ASIF e Winquist. Acta Ortop Bras. 2010;18(4):197-9 [citado 8 maio 2012]. Disponível em: http://www.scielo.br/scielo.php?pid=S1413$78522010000400004 \&$ script=sci_abstract\&tIng=pt.

3. Fraturas da diáfise femoral [citado 8 maio 2012]. Disponível em: http://www.wgate.com.br/conteudo/ medicinaesaude/fisioterapia/traumato/fratura_diafise_femoral.htm a discrepância é nas extremidades inferiores, ele é conhecido como discrepância de comprimento da perna $(\mathrm{DCP})^{19}$.

A análise crítica da metodologia utilizada nesses trabalhos, por sua vez, demonstra que a maioria deles é retrospectiva e baseada em séries de casos, razão pela qual confere-se a eles baixo poder de evidência científica.

\section{CONSIDERAÇÕES FINAIS}

Com a epidemiologia do estudo apresentou, podemos ver que anisomelia é a complicação mais freqüente e para amenizar esta complicação foram desenvolvidos parafusos bloqueantes na haste intramedular para impedir o seu desvio rotacional e diminuindo esta complicação,porem podemos notar que de acordo com o trabalho apresentado, esta complicação ainda segue disparado em primeiro lugar, nos fazendo a pensar que nem sempre os parafusos bloqueantes são utilizados, no século passado a infecção (osteomelite) era a complicação mais freqüente neste tipo de procedimento, devido as melhoras no ambiente intra-hospitalar cirúrgico, uma melhor higienização houve um descenso desta complicação, mas hoje em dia alem da infecção (osteomelite), a embolia gordurosa e a insuficiência respiratória são as mais temidas.

Um estudo mais detalhado das técnicas cirúrgicas empregadas e os fatores de risco relacionado ao procedimento podem ser úteis para uma maior diminuição das complicações do uso de haste intramedular em fratura de fêmur.

4. Paschoal FM, Paccola CAJ. Haste bloqueada "Faculdade de Medicina de Ribeirão Preto": experiência clínica no tratamento das fraturas femorais. Acta Ortop Bras. 2000;8(4) [citado 8 maio 2012]. Disponível em: http://www.scielo.br/scielo.php?script=sci_ arttext\&pid $=$ S1413-78522000000400002 \&lng=pt\&nrm=iso.

5. Reis FB. O emprego de hastes intramedulares bloqueadas no tratamento das fraturas diafisárias do úmero. Acta Ortop Bras. 2005;13(3) [citado 9 maio 2012]. Disponível em: www.scielo.br/pdf/aob/ v13n3/25673.pdf.

6. Oliveira LP, Mathias JCR, Guimarães JAM. Haste intramedular bloqueada: descrição de técnica de bloqueio distal do fêmur. Rev Bras Ortop. 1994;29(7) [citado 7 maio 2012]. Disponível em: http://www.por- 
talsaudebrasil.com/artigospsb/traum076.pdf.

7. Nelson AN, Romero ABL, Kodi EK, Bruno LC, José SHN, MTM; Ralph WC, José OSH. Resultados do tratamento das fraturas da diáfise do fêmur ipsilaterais às do colo ou transtrocantérica: Rev Bras Ortop. 2010;18(5) [citado 15 maio 2012]. Disponível em: http://www.scielo.br/scielo.php?pid=S141378522010000500004\&script=sci_arttext .

8. Pires RES, Fernandes HJA, Belloti JC, Balbachevsky D, Faloppa F, Reis FB. Como são tratadas as fraturas diafisárias fechadas do fêmur no Brasil? Estudo transversal. Acta Ortop Bras. 2006;14(3) [citado 8 maio 2012]. Disponível em: http://www.scielo.br/ scielo.php?script=sci_arttext\&pid=S1413-78522006 000300010\&lng=en\&nrm=iso\&tlng=pt.

9. Odael SJ; Luciano BR;Wellington TVC; Deliene OM; Cyril ADM; Cláudio GC; Geraldo ESA; Estevam BLC; Rafael RF. Sistemas osso-implante ex vivo utilizando haste intramedular polimérica para imobilização de fraturas femorais em bovinos jovens. 2006 fev. [citado 15 jun. 2012]. Disponível em: http://www.scielo.br/scielo.php?pid=S010384782011000200020\&script=sci_arttext

10. Astur DC, Arliani GG, Nascimento CLS, Blumetti FC, Fonseca MJA, Dobashi ET, et al. Correlação entre os índices de necrose e a estabilização precoce nas fraturas da extremidade proximal do fêmur na infância. Rev Bras Ortop. 2010;45(4) [8 maio 2012]. Disponível em: http://www.scielo.br/scielo. php?script=sci_arttext\&pid=S0102-3616201000040 0015\&lng=en\&nrm=iso\&tlng=pt.

11. Janovsky CE. Fixação intramedular das fraturas do fêmur na infância e na adolescência com hastes flexíveis. Rev Bras Ortop. 2011;46(4) [citado 07 maio 2012]. Disponível em: http://www. scielo.br/scielo.php?script=sci_arttext\&pid=S010236162011001000008\&tlng=

12. Ferracini AM. Estudo prospectivo e randomizado de pacientes com fraturas expostas da diáfise do fêmur submetidos à osteossíntese com placa e haste intramedular bloqueada a foco aberto [citado 07 maio 2012] Disponível em: http://www. scielo.br/scielo.php?script=sci_arttext\&pid=S1413$78522008000200008 \&$ Ing=en\&nrm=iso.

13. Deakin DE, Winter $\mathrm{H}$, Jain $\mathrm{P}$, Bache $\mathrm{CE}$. Malunion following flexible intramedullary nails for tibial and femoral fractures in adolescents. J Child Orthop. 2010;4(6):571-7 [cited 2012 May 7]. Available from: http://www.ncbi.nlm.nih.gov/pmc/articles/ PMC2981706/?tool=pubmed.

14. Lohiya R, Bachhal V, Khan U, Kumar D, Vijayvargiya $\mathrm{V}$, Sankhala SS, et al. Flexible intramedullary nailing in paediatric femoral fractures. A report of 73 cases. J Orthop Surg Res. 2011;6:64 [cited 2012 May 7]. Available from: http://www.ncbi.nlm.nih.gov/pmc/ articles/PMC3320542/?tool=pubmed.

15. Silva AGP. Infecção pós-estabilização intramedular das fraturas diafisárias dos membros inferiores: protocolo de tratamento. Acta Ortop Bras. 2008;16(5) [citado 8 maio 2012]. Disponível em: http://www. scielo.br/scielo.php?script=sci_arttext\&pid=S1413$78522008000500002 \&$ Ing=en\&nrm=iso.

16. Hernandezl AJ, Fávaroll E, LarayallI MHF, Zumiottil AV. Fratura espontânea do colo do fêmur após retirada de PFN. Acta Ortop Bras. 2009;17(3) [citado 8 maio 2012]. Disponível em: http://www. scielo.br/scielo.php?script=sci_arttext\&pid=S141378522009000300012.

17. Arliani GG. Correlação entre tempo para o tratamento cirúrgico e mortalidade em pacientes idosos com fratura da extremidade proximal do fêmur. Rev Bras Ortop. 2006;46(2):189-94 [citado 8 maio 2012]. Disponível em: http://www.scielo.br/scielo. php?pid=S0102-36162011000200013\&script=sci abstract\&tlng=pt.

18. Bergamaschi JPM. Análise de fraturas diafisárias do fêmur em crianças menores de 3 anos de idade. Acta Ortop Bras. 2007;15(2):72-5 [citado 9 maio 2012]. Disponível em: http://dx.doi.org/10.1590/S141378522007000200002.

19. Camile D. Concordância e correlação entre testes indiretos para avaliação de torção pélvica. Novo Hamburgo: Universidade Feevale; 2011 [citado 01 dez. 2012] Disponível em: http://ged.feevale.br/ bibvirtual/Monografia/MonografiaCamileDemboski. pdf

20. Morelli RSS, Castro ACB, Assis MC, Fernandes MS. Considerações sobre os tratamentos das fraturas de fêmur com haste intramedular de Kuntcher. Rev Bras Ortop. 1993;28:499-503 [citado 01 dez. 2012] Disponível em: http://portalsaudebrasil.com/ artigospsb/traum031.pdf 